\title{
El Valle de Colima: nuevas dataciones arqueomagnéticas para el periodo Clásico
}

\author{
Ma. Ángeles Olay Barrientos, Ligia Sofia Sánchez Morton, Avto Gogichaishvili, Juan Morales
}

\section{Ma. Ángeles Olay Barrientos}

aolayb@yahoo.com

Centro INAH Colima, Paseo de las Palapas 78 Las Palmas, Colima, 2807 Colima, México.

\section{Ligia Sofía Sánchez Morton}

Posgrado de Estudios Mesoamericanos Calle Cinco 93, Col. Espartaco, Coyoacán 04870, CDMX, México.

\section{Avto Gogichaishvili}

Juan Morales

Servicio Arqueomagnético Nacional, UNAM Campus Morelia, Antigua Carretera a Pátzcuaro No. 8701 Col. Ex-Hacienda De San José De La Huerta, 58190 Morelia, Michoacán, México.
BOL. SOC. GEOL. MEX. 2019

VOL. 71 NO. 2

P. $413-428$

http://dx.doi.org/10.18268/BSGM2019v7 ln2a11

\section{RESUMEN}

Las investigaciones arqueológicas realizadas en el Valle de Colima en los últimos años han tenido como una de sus principales limitantes la falta de una secuencia cronológica sustentada en dataciones absolutas. A partir de una revisión de la cronología propuesta por Isabel Kelly, el presente escrito da cuenta de los esfuerzos enfocados en comenzar la construcción de dicha secuencia, mediante el estudio arqueomagnético de materiales procedentes de contextos primarios excavados en tres sitios, cuya particularidad es la presencia de plazas de planta circular. El estudio aplicado a tiestos cerámicos y fragmentos de bajareque quemado ha permitido obtener las primeras dataciones absolutas para este tipo de sitios, ubicándolos dentro de lo que se conoce como el periodo Clásico mesoamericano.

Palabras clave: Valle de Colima, plazas de planta circular, arqueomagnetismo, dataciones absolutas, periodo Clásico.

\section{ABSTRACT}

The archaeological investigations carried out in the Valley of Colima in recent years have, as one of its main limitations, the lack of a chronological sequence, based on absolute dating. Based on a review of the chronology proposed by Isabel Kelly, the present work describes the efforts focused on beginning the construction of the sequence, through the archaeological-magnetic study of materials from primary contexts excavated in three sites, whose shared characteristic is the presence of circular plazas. The study of ceramic sherds and burnt bajareque fragments has allowed to obtain the first absolute dates for this type of sites, placing them within what is known as the Mesoamerican Classic period.

Keyzords: Colima Valley, circular plazas, archaeomagnetism, absolute dating, Classic period. 


\section{Introducción}

Buena parte de las investigaciones arqueológicas en el Occidente Mesoamericano han carecido de continuidad, propiciando que el conocimiento que se tiene de sus regiones sea fragmentario, y que incluso, mantengan secuencias temporales elaboradas hace ya varias décadas. Este sería el caso del Valle de Colima, en el cual se aprecia una ausencia de dataciones absolutas que otorguen certeza, tanto a la ubicación cronológica de sus secuencias cerámicas, como a las diversas variantes de asentamientos y estilos arquitectónicos asociados.

El Proyecto Arqueológico Comala se ha enfocado al estudio del sitio Comala-Potrero de la Cruz, así como de otros asentamientos en los cuales se aprecia la existencia de plazas de planta circular. En un esfuerzo por sustentar con fechas absolutas la secuencia cultural del Valle de Colima, y ante la dificultad de ubicar carbón vegetal asociado a contextos arqueológicos confiables, se intentó datar contextos mediante la extracción de colágeno residual de muestras óseas, sin obtener resultados debido a la escaza cantidad de colágeno conservado. Es por ello que hacia 2016 se consideró la pertinencia de llevar a cabo estudios de arqueomagnetismo y arqueointensidad que se presentan en este escrito, destinados a procurar fechas confiables de los sitios con evidencias de arquitectura circular.

\section{Antecedentes}

A partir de los reconocimientos efectuados en el área dominada del Volcán de Tequila, Phil C. Weigand (1985, 1993, 1996, 2008) construyó la teoría de que los grupos humanos organizados en grandes asentamientos con arquitectura circular debieran considerarse como la expresión compleja de las sociedades adscritas a la tradición de tumbas de tiro. En otras palabras, lo que este autor nombró como tradición Teuchitlán tendría que considerarse como el arquetipo del periodo Clásico en el Occidente mesoamericano.

\subsection{LA ARQUITEGTURA DE PLANTA GIRGULAR EN EL VALLE DE COLIMA}

Como parte de las labores del Proyecto Arqueológico Comala, ha sido posible registrar cuatro sitios con plazas circulares en el valle de Colima (Figura $1)$ :

- Comala: en el sitio se aprecian tres círculos de tamaños diversos, el mayor (círculo A), presenta un diámetro variable que va de los 200 a los $230 \mathrm{~m}$ (Olay y Sánchez, 2015; Figura 2).

- El sitio Potrerillos: se registraron seis círculos y una plataforma elevada; el mayor, el círculo A, tuvo un diámetro de 280 m (Olay y Sánchez, 2015; Figura 3).

- El sitio Puente de Ladrillo: solo se pudieron localizar dos círculos, ambos en franco proceso de desaparición; el círculo mayor contó con un diámetro máximo de aproximadamente $150 \mathrm{~m}$ y con plataformas perimetrales de entre 1 y $2.5 \mathrm{~m}$ de atura (Olay y Sánchez, 2015; Figura 4).

- La Herradura, explorado a partir de un salvamento arqueológico, es un sitio que presentó plataformas bajas de planta rectangular organizadas alrededor de una plaza semi circular, edificada sobre la ladera de una loma de $12 \mathrm{~m}$ de alto (Olay et al., 2007; Figura 5).

\subsection{VALIDEZ Y VIGENGIA DE LA SECUENGIA GULTURAL DEL EJE ARMERÍA}

Glenn Stuart (2003) es el primero en reportar nueve fechas de radiocarbón. A partir de los trabajos en el sitio Guachimontones se obtuvieron otras 18. La exploración por parte de Christopher Beekman (2000) en el sitio Llano Grande procuró otras 38 fechas, a las que se sumaron 29 más obtenidas en las exploraciones en el sitio de Navajas 


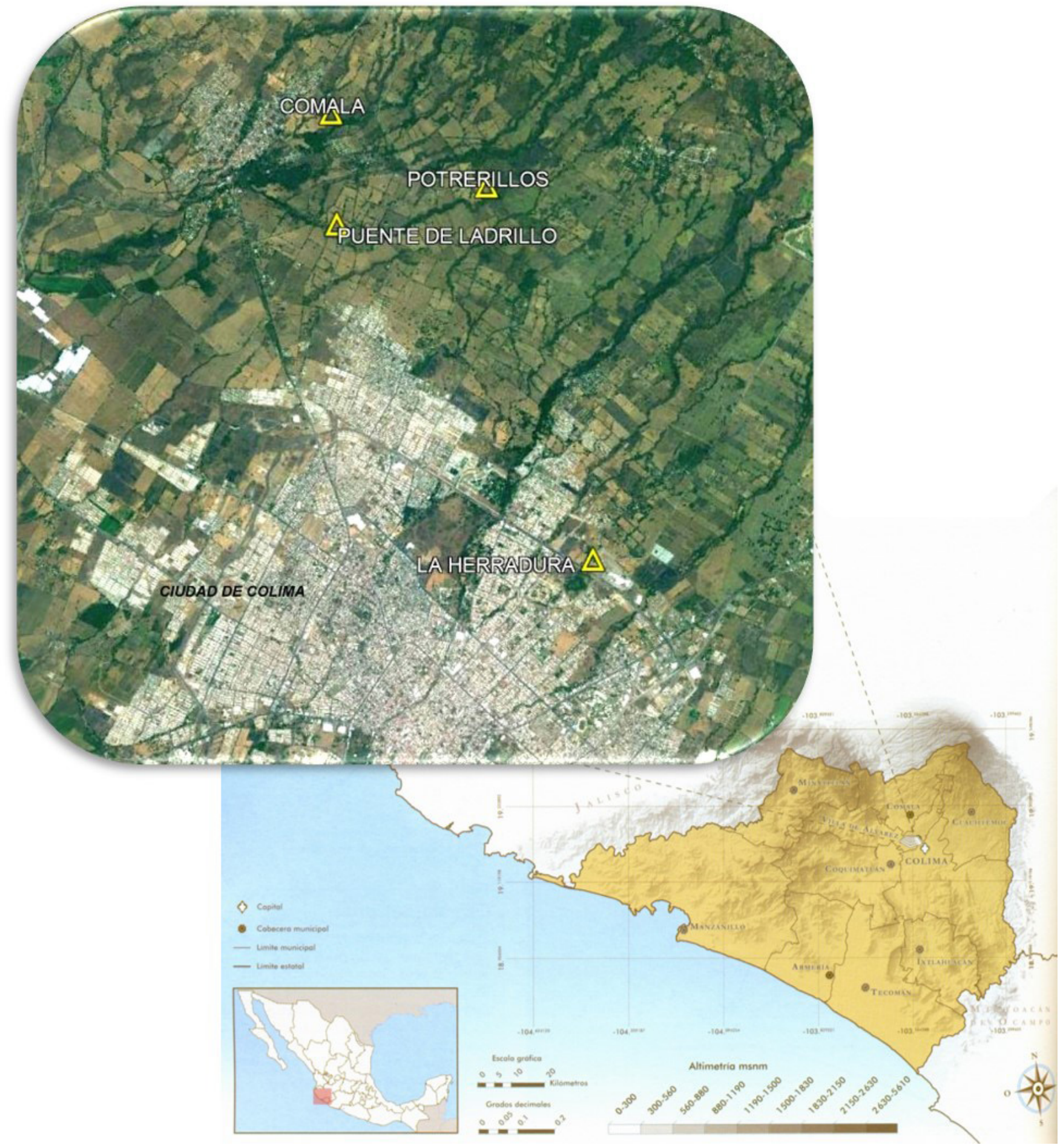




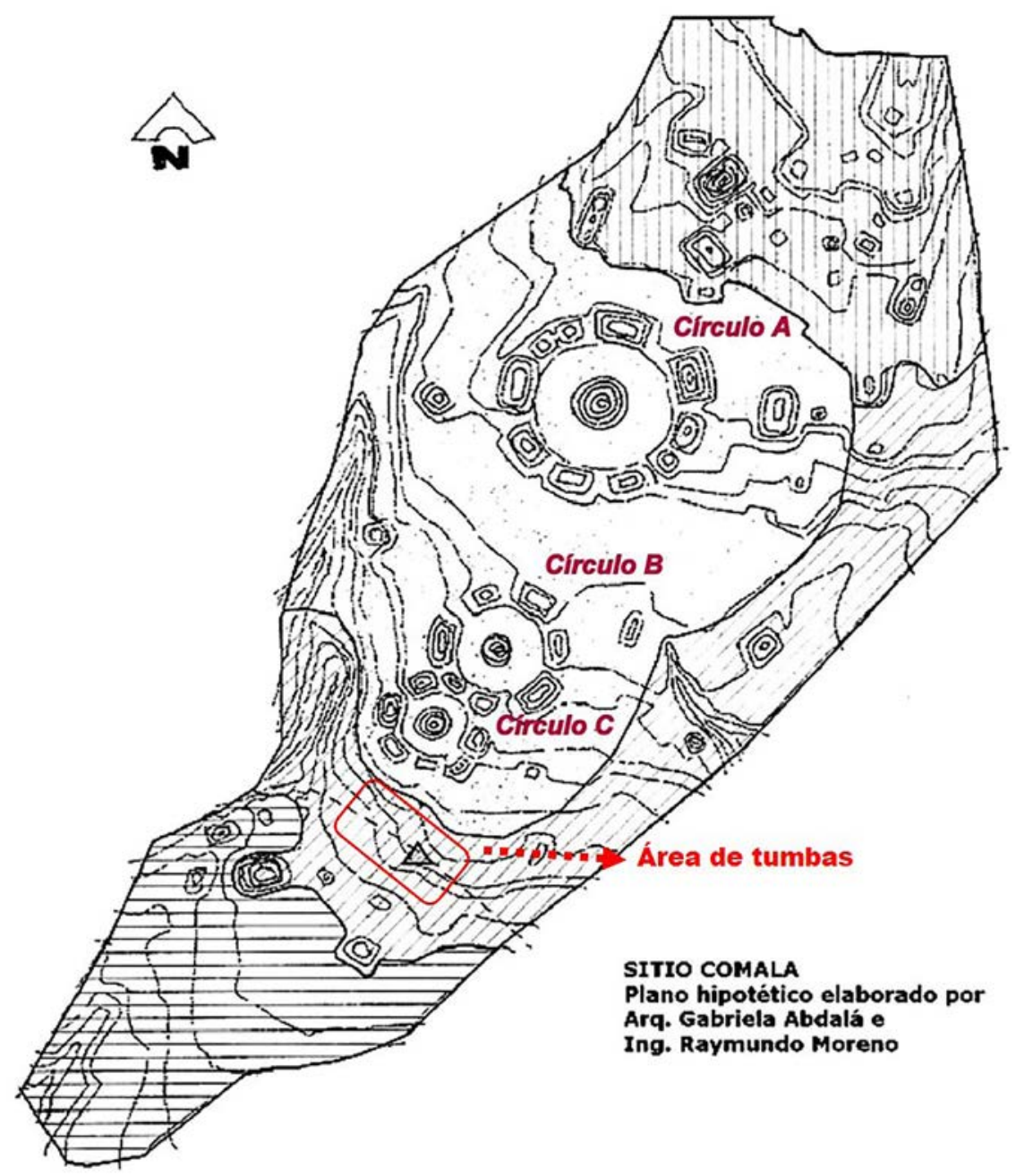

Figura 2 Mapa del sitio Comala y los círculos al interior. Retomado de Gabriela Abdalá (2006); las muestras proceden del Círculo A y del área de tumbas.

(Weigand y Beekman, 2008: 307-308)). Finalmente, no se deben pasar por alto las 16 fechas más obtenidas por Verónica López (2016) en los sitios Guachimontones, Loma Alta y La Joyita A y B. En total estamos hablando de 120 fechas de radiocarbono destinadas a verificar o negar, en su caso y con datos duros, las hipótesis inicialmente planteadas por Phil C. Weigand.

Para el caso de Colima, los primeros reconocimientos y exploraciones arqueológicas efectuados por Kelly entre 1939 y 1940, dieron como resultado el registro de alrededor de 20 sitios en la cuenca del río Salado (Kelly, 1989: 71-73; Figura 6), y se concretaron en su conocida obra sobre la fase Capacha y la secuencia cerámica de Colima (Kelly, 1980; Tabla 1); la secuencia soportada con 15 fechas de radiocarbón, permitió contextualizar el devenir cultural del área central de Colima (Valle de Colima y cuenca baja del río Armería), a la cual denominó como Eje Armería.

Las fechas reportadas para los materiales obtenidos por Kelly han provocado numerosas discusiones relativas a su pertinencia y a la baja confiabilidad de estas, tanto por proceder de con- 
textos de removidos o sujetos de saqueo, así como por las problemáticas inherentes a las dataciones previas a la aplicación de los estándares actuales: la falta de calibraciones, que no se considerara la edad reservorio para las muestras de concha o el método empleado para obtener la edad calendario, entre otros (Olsson, 2009).

No obstante, el posterior hallazgo de varios contextos tempranos en el valle de Colima (Alcántara, 2005; Alcántara y Galicia, 2008; Almendros, 2012), han sido pocos los esfuerzos encaminados en convalidar las fechas propuestas por Kelly hacia 1980; uno de los más relevantes es la datación por medio de arqueointensidad de siete fragmentos de cerámicas Capacha, lo que dio como resultado un intervalo cronológico entre 1481 a 1256 aC, que es congruente con lo planteado por Kelly (Morales et al., 2013).

\section{Selección de materiales para este estudio}

La selección de materiales para estos estudios incluyó tiestos cerámicos, fragmentos de bajareque quemado y fragmentos de piso de arcilla quemado (Tabla 2).

Dado que el piso pudo ser recuperado con dirección magnética, fue posible datarlo mediante arqueomagnetismo: el resultado obtenido fue un

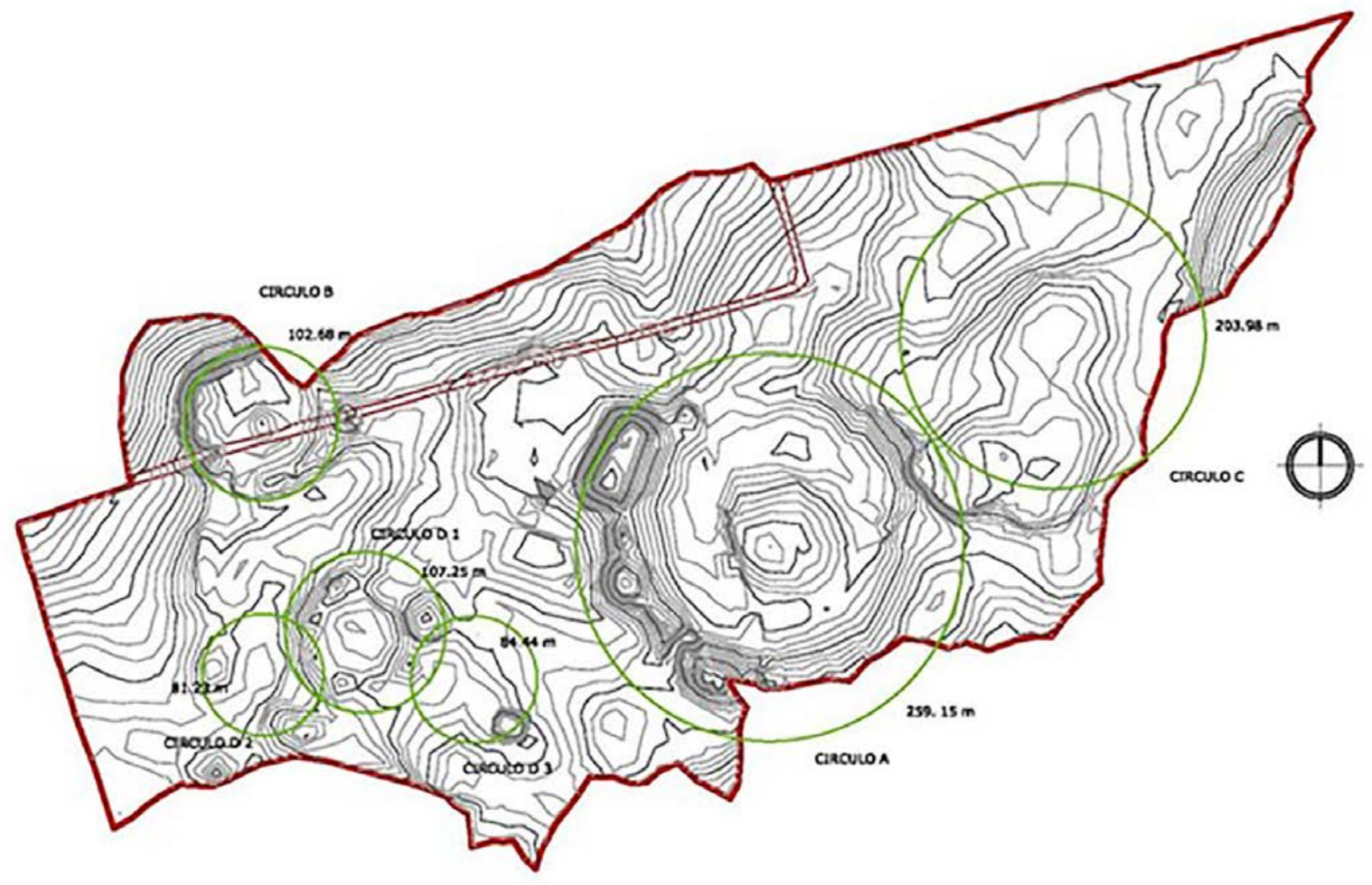




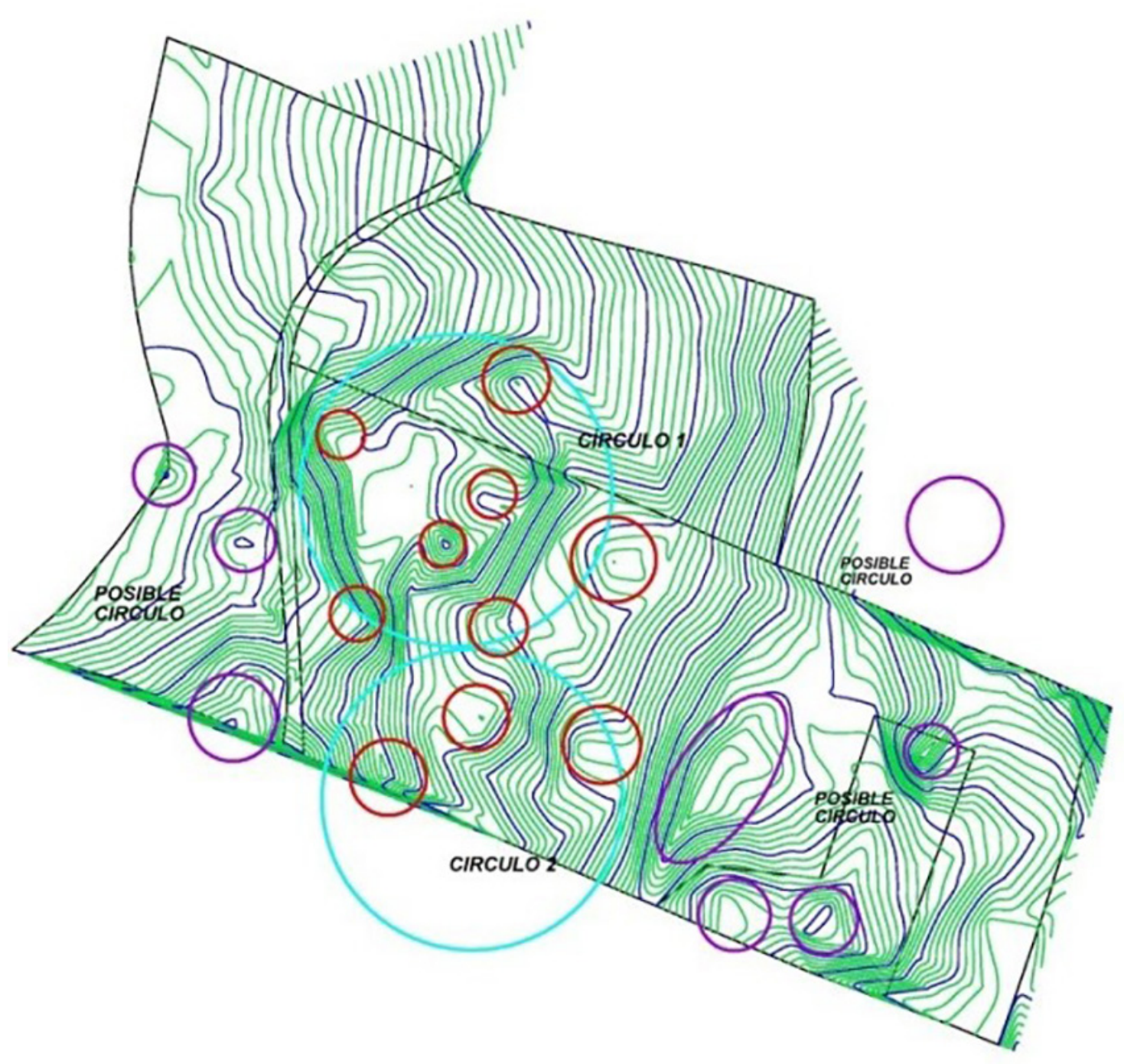

Figura 4 Plano topográfico del sitio Puente de Ladrillo, con ubicación de los Círculos 1 y 2; las muestras se recuperaron al interior del Círculo 1 (Olay y Sánchez, 2015).

intervalo cronológico de 548-585 dC, mismo que ubicaría el contexto hacia el fin de la fase Comala, momento que marca el inicio de las grandes transformaciones y el devenir de las sucesivas poblaciones que habitaron en el Valle de Colima hacia el Clásico tardío (650 a 900 dG).

Si bien esta fecha solo permitió datar un contexto de combustión muy específico, y no permitía hacer planteamientos de orden más general sobre la ocupación de sitio, se consideró que la datación de tiestos cerámicos por métodos de arqueointensidad sería una alternativa que permitiría el objetivo de construir la secuencia cronológica de los sitios. De forma tal se seleccionaron 13 fragmentos cerámicos de diversos contextos pertenecientes a los sitos explorados (Tabla 3):

- Contextos en el sitio Comala: a fin de establecer con mayor claridad el momento en el cual se edificaron y funcionaron los elementos arquitectónicos del Círculo A del sitio, se seleccionaron seis 


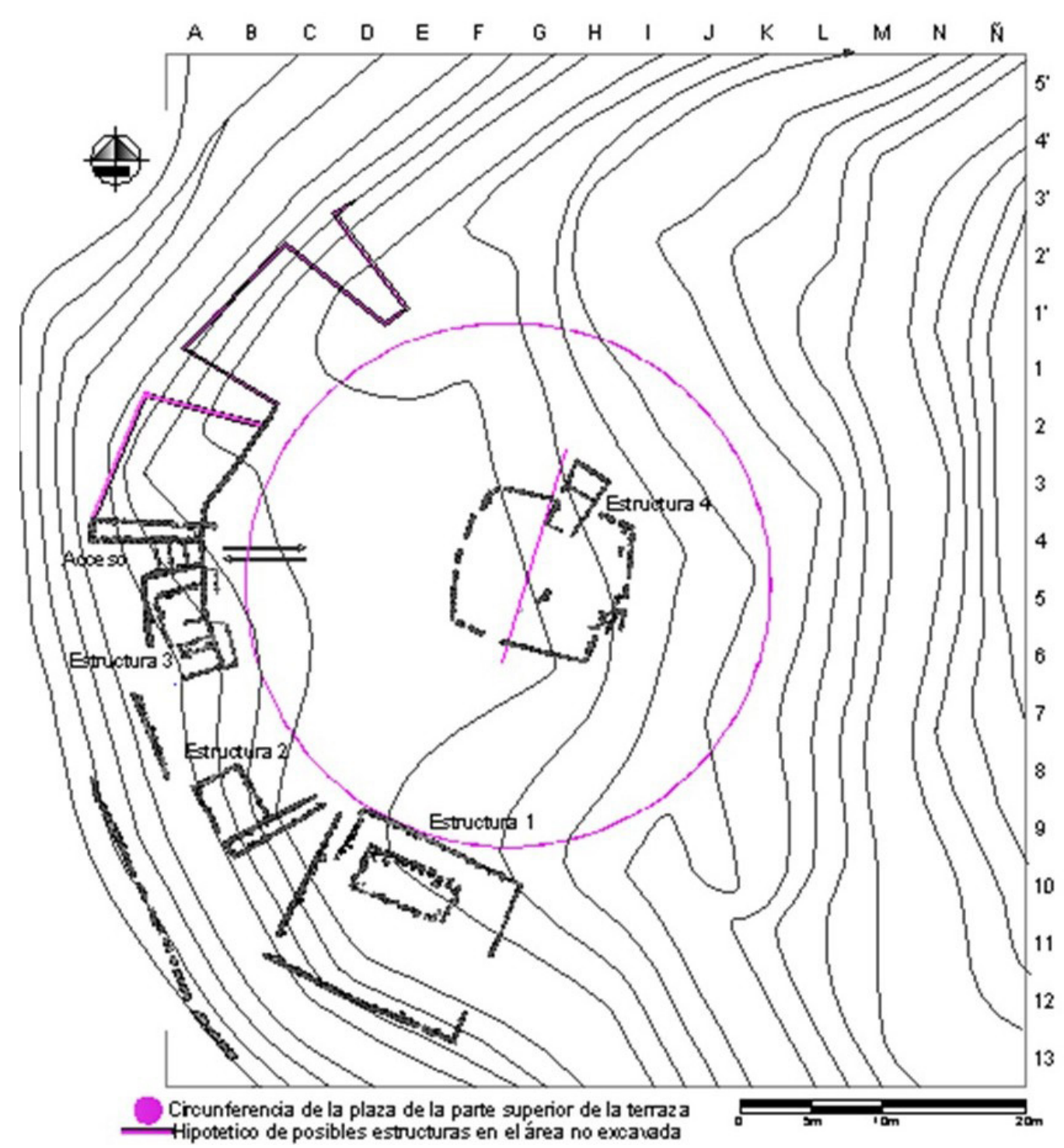

fragmentos cerámicos y un fragmento de arcilla quemada, procedentes de las exploraciones realizadas durante las temporadas 2010 y 2011; en el caso de los tiestos cerámicos, se seleccionaron tres cuya identificación tipológica los asociaba a la fase Ortices, y tres a la fase Comala; adicionalmente se seleccionaron dos fragmentos de bajareque quemado, procedentes de contextos excavados en la llamada área de tumbas del sitio, explorada como parte del Salvamento Juan Silva Palacios (Figura 2).
- Contextos en el sitio Puente de Ladrillo: las excavaciones al interior del Círculo 1 del sitio permitieron recuperar tiestos al interior de las capas consideradas como relleno estructural; se seleccionaron cuatro fragmentos cerámicos, dos identificados como tipos de la fase Ortices y dos de la fase Comala, así como un fragmento de bajareque quemado (Figura 4).

- Contextos en el sito La Herradura: se eligió una muestra de bajareque quemado procedente de la exploración realizada en la estructura central de 
la plaza, en un contexto asociado a restos óseos de dos mujeres, acompañadas de ofrendas consistentes en vasijas y manos de metate; materiales que remitían de manera clara a las fases Ortices y Comala (Figura 5).

\section{Estudio arqueomagnético}

Los componentes del campo geomagnético (declinación, inclinación e intensidad) son medibles en rocas y materiales arqueológicos, siempre y cuando sea posible obtener su orientación in situ, sin embargo, aquellos con magnetismo termoremanente (TRM), como la cerámica, permiten medir la magnitud del vector, sin la necesidad de conocer su orientación, ya que teóricamente se ha propuesto que la intensidad de la magnetización termoremanente es linealmente proporcional a la intensidad del campo adquirido cuando la cerámica adquirió dicha termoremanencia (Sternberg, 1990: 22-23).

La selección de las muestras con mayor confiabilidad para las determinaciones de intensidad absoluta geomagnética se basó en los experimentos del registro de la susceptibilidad magnética en función

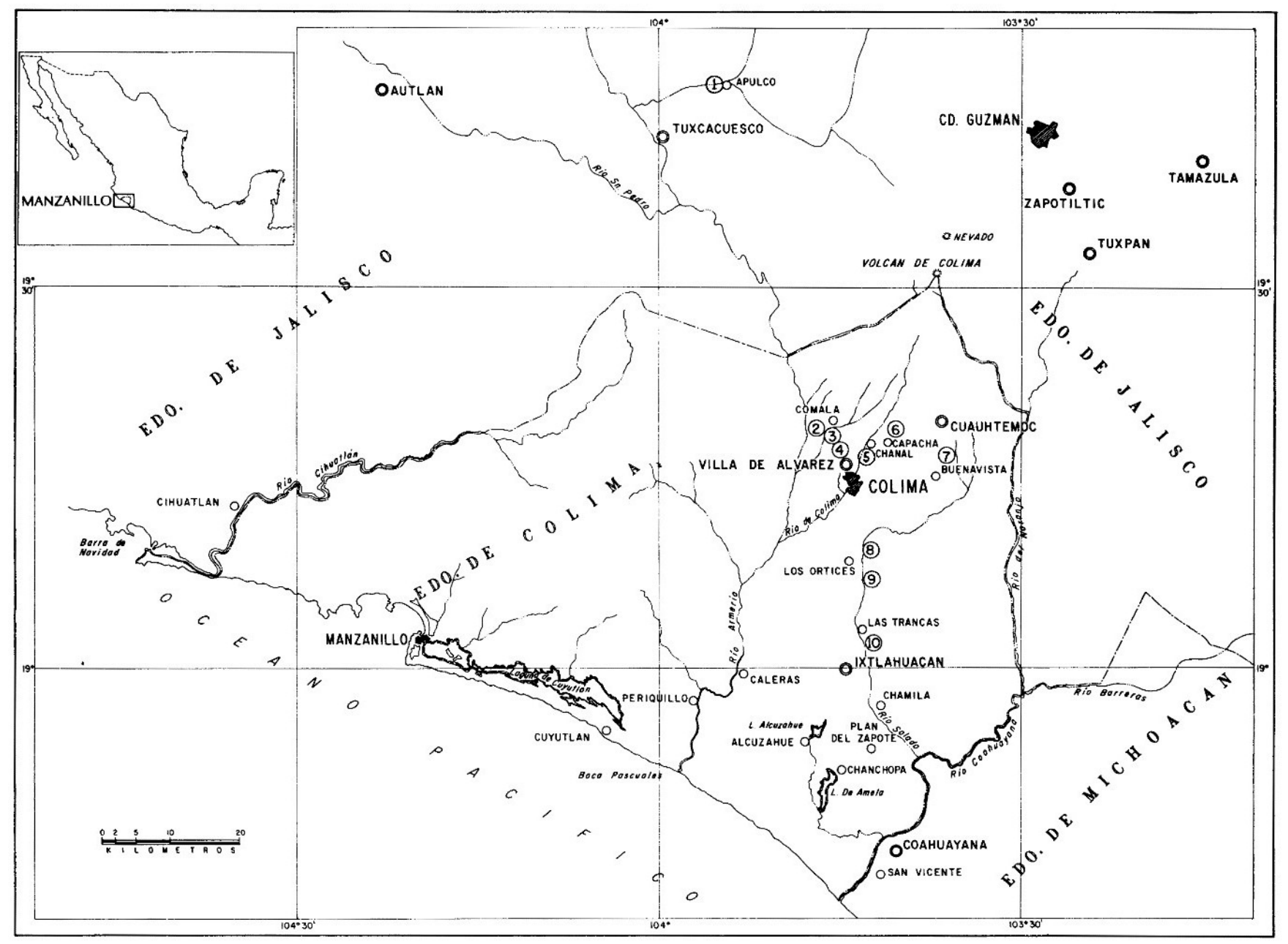

Mapa de Colima que muestra los sitios explorados por Isabel Kelly (1980: 2). 
Tabla 1. Fechas de radiocarbono para diversas fases de Colima (Kelly, 1980: 4).

\begin{tabular}{|c|c|c|c|c|c|c|c|c|}
\hline No. & $\begin{array}{c}\text { Fase } \\
\text { Cerámica }\end{array}$ & $\begin{array}{c}\text { Número de } \\
\text { identificación } \\
\text { del laboratorio }\end{array}$ & Material & $\begin{array}{c}\text { Edad C-14 } \\
\text { (B.P.) }\end{array}$ & $\begin{array}{l}\text { Edad C-14 } \\
\text { (A.D./B.C.) }\end{array}$ & $\begin{array}{c}\text { Ajuste a } 5730 \\
\text { años de vida } \\
\text { media }\end{array}$ & $\begin{array}{c}\text { Ajuste al } \\
\text { Tiempo } \\
\text { Astronómico }\end{array}$ & Procedencias \\
\hline 1 & Periquillo & UCLA-1095-C & Caracol & $325 \pm 60$ & $\begin{array}{l}\text { A.D. } 1600 \mathrm{o} \\
\text { A.D. } 1450\end{array}$ & A.D. 1615 & $\begin{array}{l}\text { A.D. } 1520 \\
\text { (A.D. } 1440- \\
1630)\end{array}$ & $\begin{array}{c}\text { El Columpio, Prueba } 2: 3 \\
\text { (30-45 cm bajo la } \\
\text { superficie })\end{array}$ \\
\hline 2 & Chanal & $\begin{array}{l}\text { M-2338 (Crane } \\
\text { y Griffin 1972: } \\
184)\end{array}$ & Caracol & $660 \pm 100$ & A.D. 1290 & A.D. 1270 & $\begin{array}{l}\text { A.D. } 1260- \\
1290 \text { (A.D. } \\
1200-1380)\end{array}$ & $\begin{array}{c}\text { El Chanal Este, Prueba } 3: 5 \\
\text { (80-100 cm bajo la } \\
\text { superficie) }\end{array}$ \\
\hline 3 & Chanal & $\begin{array}{l}\text { M-2334 (Crane } \\
\text { y Griffin 1972: } \\
184)\end{array}$ & Caracol & $490 \pm 100$ & A.D. 1460 & A.D. 1445 & $\begin{array}{l}\text { A.D. } 1410 \\
\text { (A.D. } 1340- \\
1450)\end{array}$ & $\begin{array}{c}\text { El Chanal Oeste, Potrero } \\
\text { de los Edificios (chicos), } \\
\text { prueba } 1 \text { excavación } \\
\text { general }\end{array}$ \\
\hline 4 & Armería & $\begin{array}{l}\text { M-2339 (Crane } \\
\text { y Griffin 1972: } \\
184)\end{array}$ & Concha & $1260 \pm 130$ & A.D. 690 & A.D. 652 & $\begin{array}{l}\text { A.D. } 690 \text { (A.D. } \\
590-880)\end{array}$ & $\begin{array}{c}\text { El Bajadero, Las Borregas, } \\
\text { Entierro } 11\end{array}$ \\
\hline 5 & Colima & UCLA-1095-A & Caracol & $1440 \pm 60$ & A.D. 510 & A.D. 467 & $\begin{array}{l}\text { A.D. } 557 \text { (A.D. } \\
460-610)\end{array}$ & $\begin{array}{l}\text { Potrero del Rancho Nuevo } \\
\text { No. 3, Los Asmoles; Pozo } \\
\text { de prueba, corte } 5 \text { ( } 90-100 \\
\text { bajo superficie. Sobre } \\
\text { arena estéril.) }\end{array}$ \\
\hline 6 & Colima (?) & UCLA-1651 & $\begin{array}{l}\text { Hueso } \\
\text { Humano }\end{array}$ & $<500$ años & & & & $\begin{array}{c}\text { La Parranda (Sitio A) } \\
\text { Entierro } 1\end{array}$ \\
\hline 7 & Comala (?) & $\begin{array}{l}\text { M-2339 (Crane } \\
\text { y Griffin 1972: } \\
183)\end{array}$ & Concha & $2350 \pm 140$ & 400 B.C. & 470 B.C. & $\begin{array}{l}\text { 460-440 B.C. } \\
\text { (765-395 B.C.) }\end{array}$ & $\begin{array}{c}\text { Tumba Compilada } \\
\text { Comprada, Rancho del } \\
\text { Escritorio Tuxpan (Jalisco) }\end{array}$ \\
\hline 8 & $\begin{array}{l}\text { Comala (?) } \\
\text { Ortices (?) }\end{array}$ & UCLA-1095-B & Caracol & $1450 \pm 60$ & A.D. 500 & A.D. 456 & $\begin{array}{c}\text { A.D. } 545 \text { (A.D. } \\
460-600)\end{array}$ & $\begin{array}{l}\text { Las Lomas, Los Asmoles } \\
\text { "horno", 40-60cm bajo la } \\
\text { superficie sobre arena } \\
\text { estéril }\end{array}$ \\
\hline 9 & Comala (?) & UCLA-1627 & Concha & $1360 \pm 80$ & A.D. 590 & A.D. 550 & $\begin{array}{l}\text { A.D. } 620 \text { (A.D. } \\
545-690)\end{array}$ & $\begin{array}{c}\text { Tumba Compilada } \\
\text { Comprada, "Tierra de } \\
\text { Solórzano", Los Orices. } \\
\text { Ppresenta asociación con } \\
\text { cerámica Amoles wiped }\end{array}$ \\
\hline 10 & Ortices (?) & UCLA-1066 & Concha & $2180 \pm 80$ & 230 B.C. & 295 B.C. & $\begin{array}{l}\text { 290-270 B.C. } \\
\text { (420-140 B.C.) }\end{array}$ & $\begin{array}{l}\text { Chanchopa sitio } 2 . \\
\text { Procedente de tumba } \\
\text { saqueada }\end{array}$ \\
\hline 11 & Ortices & $\begin{array}{c}\text { M-2396 (Crane } \\
\text { y Griffin 1972: } \\
185)\end{array}$ & Caracol & $2110 \pm 140$ & 160 B.C. & 223 B.C. & $\begin{array}{l}\text { 200-170 B.C. } \\
(410 \text { B.C.-A.D. } \\
50)\end{array}$ & $\begin{array}{l}\text { La Paranera, San Vicente, } \\
\text { Valle del Coahuayana, } \\
\text { Michoacán. Pozo de } \\
\text { prueba este, } 340 \mathrm{~cm} \text { bajo } \\
\text { superficie }\end{array}$ \\
\hline 12 & Ortices & $\begin{array}{c}\text { M-2341 A } \\
\text { (Crane y Griffin } \\
\text { 1972: 184) }\end{array}$ & Concha & $1690 \pm 140$ & A.D. 260 & A.D. 211 & $\begin{array}{c}\text { A.D. } 270 \text { (A.D. } \\
134-440)\end{array}$ & $\begin{array}{l}\text { Loma del Volantín, } \\
\text { Alcuzahue tumba removide }\end{array}$ \\
\hline 13 & Ortices & $\begin{array}{c}\text { M-2341 B } \\
\text { (Crane y Griffin } \\
\text { 1972: 184-185) }\end{array}$ & Concha & $1330 \pm 140$ & 280 B.C. & 347 B.C. & $\begin{array}{l}410 \text { B.C. }(480- \\
140 \text { B.C. })\end{array}$ & $\begin{array}{c}\text { Misma tumba removida de } \\
\text { número anterior }\end{array}$ \\
\hline 14 & Copacha & GX-1784 & Tiestos & $3400 \pm 200$ & 1450 B.C. & 1552 B.C. & $\begin{array}{c}\text { 1870-1720 B.C. } \\
(2110-1520 \\
\text { B.C. })\end{array}$ & $\begin{array}{c}\text { Terreno de Jesús } \\
\text { Gutiérrez, Villa de } \\
\text { Álvarez; superficie, } \\
\text { recolectado por monero } \\
\text { procedente de entierros } \\
\text { saqueados }\end{array}$ \\
\hline 15 & Copacha & $\begin{array}{l}\text { UCR-129; } \\
\text { revisado entre } \\
\text { laboratorios } \\
\text { UCLA-1888 }\end{array}$ & Caracol & $180 \pm 100$ & A.D. 1770 & A.D. 1765 & $\begin{array}{l}\text { A.D. } 1625 \\
\text { (A.D. } 1520- \\
1880)\end{array}$ & $\begin{array}{l}\text { Quintero; debajo de } \\
\text { "horno" excavado por } \\
\text { moneros; obviamente } \\
\text { contaminado }\end{array}$ \\
\hline
\end{tabular}


de temperatura, y el tratamiento magnético por medio de campos alternos por etapas desde $5 \mathrm{mT}$; los resultados descartaron 6 de las 13 muestras.

De forma tal, se preseleccionaron 7 fragmentos (4 de bajareque y 3 de cerámica), pues debido a su buen comportamiento magnético observado durante las mediciones de desmagnetización por campos alternos hasta $90 \mathrm{mT}$, se consideraron aptos para experimentos de intensidad absoluta de doble calentamiento tipo Thellier; Estos fragmentos se rompieron adicionalmente en al menos 6 piezas (42 especímenes), y se presionaron en paletas de sal para facilitar su tratamiento como muestras estándar. Los experimentos de tipo Thellier-Coe (Thellier y Thellier, 1959; Coe, 1967) se llevaron a cabo usando un desmagnetizador y remagnetizador térmico marca Magnetic Measurements Ltd.

Se aplicaron trece pasos de temperatura, los cuales se distribuyeron desde la temperatura ambiente hasta $560^{\circ} \mathrm{C}$, con reproducibilidad entre dos ciclos de calentamiento a la misma temperatura, mejor que $2^{\circ} \mathrm{C}$; la intensidad de campo del laboratorio se estableció en $(40.0 \pm 0.06) \mu \mathrm{T}$; se realizaron cuatro chequeos de control a través de las magnetizaciones termoremanentes parciales a lo largo de los experimentos; la dependencia de velocidad de enfriamiento de TRM (magnetización termoremanente por sus siglas en inglés) se realizó según un procedimiento modificado descrito en Chauvin et al. (2000; Morales et al., 2009), usando un enfriamiento lento de $6 \mathrm{~h}$ desde $560^{\circ} \mathrm{C}$, hasta la temperatura ambiente.

Los criterios de aceptación para las determinaciones individuales de arqueointensidad (Paterson, 2011) se pueden resumir de la siguiente manera:

1. Las direcciones de los puntos finales de magnetización remanente natural (NRM por sus siglas en ingles) en cada paso obtenido de los experimentos de doble calentamiento de Thellier, deben formar una línea razonablemente recta, tendiendo hacia el origen en el intervalo de temperatura elegido para la determinación de la arqueointensidad.

2. No debe observarse ninguna desviación significativa de las direcciones de NRM hacia la dirección del campo aplicado.

Tabla 2. Tipo, procedencia y asociación de las muestras empeladas para el estudio arqueomagnético.

\begin{tabular}{|c|c|c|c|c|}
\hline Muestra & Tipo & Sitio & Fase & Asociación \\
\hline M1 & Bajareque quemado & Comala & & UEE4 CIII \\
\hline M2 & Bajareque quemado & Comala & & UEE6 CII \\
\hline M3 & Bajareque quemado & Puente de Ladrillo & & Pozo 3 CI \\
\hline M4 & Bajareque quemado & La Herradura & & UEE5, C2, C11E, CII \\
\hline M5 & Arcilla quemada & Comala & & UEE5, C III \\
\hline M6 & Cerámica & Comala & Ortices & Estructura 9 C III \\
\hline M7 & Cerámica & Comala & Comala & Estructura 13 CII \\
\hline M8 & Cerámica & Comala & Ortices & Estructura 9 CI \\
\hline M9 & Cerámica & Comala & Comala & Estructura 13 CIII \\
\hline M10 & Cerámica & Puente de Ladrillo & Ortices & Patio CIII \\
\hline M11 & Cerámica & Puente de Ladrillo & Ortices & Patio CII \\
\hline M12 & Cerámica & Puente de Ladrillo & Comala & EstructuraSur C I \\
\hline M13 & Cerámica & Puente de Ladrillo & Comala & EstructuraSur C IIa \\
\hline
\end{tabular}


Tabla 3. La determinación de la intensidad absoluta geomagnética por medio de experimento de Thellier de doble calentamiento. T1-T2 refiere al intervalo de temperatura usado para la determinación de la arqueointensidad, $\mathrm{N}$ es número de etapas de ciclos de desmagnetizaciones y remagnetizaciones, $\mathrm{f}, \mathrm{g}$ y $\mathrm{q}$ son factores de calidad descritos en Coe et al. (1978). $\mathrm{H}$ son valores de arqueointensidad corregidas por el ritmo de enfriamiento.

\begin{tabular}{|c|c|c|c|c|c|c|c|c|c|}
\hline Muestra & Espécimen & Cod-lab & T1-T2 $\left({ }^{\circ} \mathrm{C}\right)$ & $\mathbf{N}$ & f & G & $q$ & $\begin{array}{c}\text { H(corr) } \\
\text { (Mt) }\end{array}$ & $\pm \mathrm{sH}$ \\
\hline \multirow{6}{*}{ M1 } & A & 99L001A & $350-540$ & 7 & 0.78 & 0.623 & 11.6 & 41.9 & 1.8 \\
\hline & B & 99L001B & $300-540$ & 8 & 0.772 & 0.592 & 10.52 & 49.7 & 2.3 \\
\hline & $\mathrm{C}$ & 99L001C & $350-560$ & 8 & 0.823 & 0.674 & 10.91 & 41.2 & 0.9 \\
\hline & D & 99L001D & $300-560$ & 9 & 0.854 & 0.727 & 6.98 & 49.6 & 2.1 \\
\hline & E & 99L001E & N.D. & & & & & & \\
\hline & $\mathrm{F}$ & 99L001F & N.D. & & & & & & \\
\hline \multirow{6}{*}{ M2 } & A & 99L002A & $300-540$ & 8 & 0.811 & 0.718 & 13.2 & 53.8 & 1.9 \\
\hline & B & 99L002B & $350-540$ & 7 & 0.823 & 0.687 & 11.61 & 52.1 & 1.8 \\
\hline & $\mathrm{C}$ & 99L002C & $350-540$ & 7 & 0.778 & 0.598 & 9.62 & 49.9 & 1.5 \\
\hline & D & 99L002D & $350-560$ & 8 & 0.784 & 0.843 & 5.96 & 51.7 & 2.1 \\
\hline & E & 99L002E & $300-560$ & 9 & 0.754 & 0.849 & 8.56 & 52.8 & 2.3 \\
\hline & $\mathrm{F}$ & 99L002F & $300-540$ & 8 & 0.885 & 0.842 & 9.32 & 50.1 & 1.7 \\
\hline \multirow{6}{*}{ M3 } & A & 99L003A & $400-540$ & 6 & 0.721 & 0.523 & 4.32 & 48.5 & 2.1 \\
\hline & B & 99L003B & $400-515$ & 5 & 0.763 & 0.456 & 5.47 & 52.6 & 2.3 \\
\hline & $\mathrm{C}$ & 99L003C & $400-540$ & 6 & 0.854 & 0.562 & 7.74 & 56.7 & 2.9 \\
\hline & D & 99L003D & N.D. & & & & & & \\
\hline & E & 99L003E & N.D. & & & & & & \\
\hline & $\mathrm{F}$ & 99L003F & N.D. & & & & & & \\
\hline \multirow{6}{*}{ M4 } & A & 99L004A & $350-540$ & 7 & 0.845 & 0.618 & 6.68 & 41.2 & 1.6 \\
\hline & B & 99L004B & $350-540$ & 7 & 0.79 & 0.623 & 7.12 & 43.7 & 1.8 \\
\hline & $\mathrm{C}$ & 99L004C & muestra & Inservible & & & & & \\
\hline & D & 99L004D & $350-515$ & 6 & 0.779 & 0.514 & 4.92 & 42.9 & 2.2 \\
\hline & E & 99L004E & N.D. & & & & & & \\
\hline & $\mathrm{F}$ & 99L004F & N.D. & & & & & & \\
\hline \multirow{6}{*}{ M8 } & A & 99L005A & $300-540$ & 8 & 0.825 & 0.654 & 8.62 & 51.6 & 2.3 \\
\hline & B & 99L005B & $300-540$ & 8 & 0.778 & 0.668 & 8.36 & 48.2 & 2 \\
\hline & $\mathrm{C}$ & 99L005C & $350-540$ & 7 & 0.795 & 0.596 & 5.64 & 53.9 & 2.1 \\
\hline & D & 99L005D & $300-540$ & 8 & 0.845 & 0.634 & 6.78 & 56.2 & 2.3 \\
\hline & E & 99L005E & $350-540$ & 7 & 0.815 & 0.547 & 6.25 & 53.3 & 1.9 \\
\hline & $\mathrm{F}$ & 99L005F & $300-540$ & 8 & 0.884 & 0.698 & 9.16 & 50.6 & 1.6 \\
\hline \multirow{6}{*}{ M11 } & A & 99L006A & $350-540$ & 7 & 0.884 & 0.672 & 11.52 & 56.1 & 2.6 \\
\hline & B & 99L006B & $350-540$ & 7 & 0.816 & 0.654 & 9.81 & 56.4 & 2.4 \\
\hline & $\mathrm{C}$ & 99L006C & $300-540$ & 8 & 0.865 & 0.745 & 12.51 & 55.8 & 3.1 \\
\hline & D & 99L006D & $300-540$ & 8 & 0.846 & 0.752 & 11.55 & 53.6 & 2.8 \\
\hline & E & 99L006E & N.D. & & & & & & \\
\hline & $\mathrm{F}$ & 99L006F & $300-560$ & 9 & 0.872 & 0.786 & 13.27 & 54.9 & 2.1 \\
\hline \multirow{6}{*}{ M13 } & A & 99L007A & $300-540$ & 8 & 0.844 & 0.715 & 7.42 & 38.7 & 1.5 \\
\hline & B & 99L007B & $350-540$ & 7 & 0.798 & 0.623 & 7.78 & 37.2 & 1.9 \\
\hline & $\mathrm{C}$ & 99L007C & $300-540$ & 8 & 0.826 & 0.81 & 9.62 & 37.1 & 1.8 \\
\hline & D & 99L007D & $350-540$ & 7 & 0.811 & 0.568 & 6.54 & 44.6 & 2.3 \\
\hline & E & 99L007E & $350-540$ & 7 & 0.785 & 0.593 & 5.68 & 33.4 & 0.9 \\
\hline & $\mathrm{F}$ & 99L007F & $300-540$ & 8 & 0.865 & 0.689 & 9.23 & 35.1 & 1.5 \\
\hline
\end{tabular}


Tabla 4. Probables intervalos de edad asignadas a cada uno de los fragmentos estudiados a base de la datación arqueomagnética usando el modelo de campo geomagnético SHA.DIF.14K de Pavon-Carrasco et al. (2014.)

\begin{tabular}{|c|c|c|c|}
\hline Muestra & Tipo & Sitio & $\begin{array}{c}\text { Fechamiento arqueomagnético } \\
\text { (SHA.DIF14K) }\end{array}$ \\
\hline M1 & Bajareque & Comala & 511-683 AD \\
\hline M2 & Bajareque & Comala & $(163-322$ AD), (400-472 AD) \\
\hline M3 & Bajareque & Puente de Ladrillo & (1058-1229 AD), (1322-1453 AD) \\
\hline M4 & Bajareque & Herradura & 68-137 AD \\
\hline M8 & Cerámica & Comala & (165-316 AD), (411-465 AD) \\
\hline M11 & Cerámica & Puente de Ladrillo & (132 BC-34 AD), (206-255 AD) \\
\hline
\end{tabular}

3. Se deben usar al menos cinco puntos TRMNRM alineados en la curva de Arai-Nagata.

4. Factor de fracción NRM f $\geq 0.3$ (Coe et al., 1978); esto significa que al menos el 30 por ciento del NRM inicial se usó para la determinación de la arqueointensidad.

5. Factor de calidad $q \geq 4$ (generalmente mayor que 5) (Coe et al., 1978).

6. Los resultados de arqueointensidad obtenidos de los diagramas NRM-pTRM no deben mostrar una forma cóncava evidente, ya que en tales casos la remanencia probablemente se asocia con la presencia de granos de MD (multidominios; Levi, 1977; Kosterov et al., 1998).

7. Chequeos de control positivos, es decir, la desviación de las verificaciones pTRM debe ser menor del 15 por ciento en comparación con el paso original NRM-TRM.

\section{Resultados}

$\mathrm{Al}$ término de estos procedimientos, 33 muestras analizadas arrojaron resultados técnicamente aceptables (tablas 3 y 4 ) bajo los criterios arriba expuestos.

Con la finalidad de realizar las estimaciones de los probables intervalos de edad, se usó el modelo geomagnético SHA.DIF.14k (Pavón-Carrasco et al., 2014), considerado el de mayor resolución en la variación del campo geomagnético en los últimos 14000 años. Se anexan los resultados de estas estimaciones en la Tabla 4; un ejemplo de la gráfica correspondiente se presenta en la Figura 7. Para hacer una determinación de la edad del artefacto arqueológico quemado, las componentes de su magnetización remanente (intensidad, declinación e inclinación) se comparan con una Curva de referencia de Variación Secular, completada por patrones generados por modelos estadísticos globales (Pavón-Carrasco et al., 2014).

\section{Análisis}

En cuanto a las estimaciones de edades para los siete fragmentos preseleccionados, es necesario considerar que, para dos muestras de bajareques (M1 y M4), así como para la cerámica del sitio Puente de Ladrillo (M13), el ejercicio de la datación arqueomagnética arroja un único intervalo de edad, y por tanto la interpretación carece de ambigüedad dentro del intervalo elegido de datación. Sin embargo, para el resto de muestras, se obtuvieron dos posibles intervalos de edad (muestras de bajareque M2 y M3 y cerámica M8 y M11), lo cual es común en el arqueomagnetismo debido a la ocurrencia de la intensidad similar de campo geomagnético para los diferentes periodos arqueológicos. En estos casos duales, la elección del inter- 

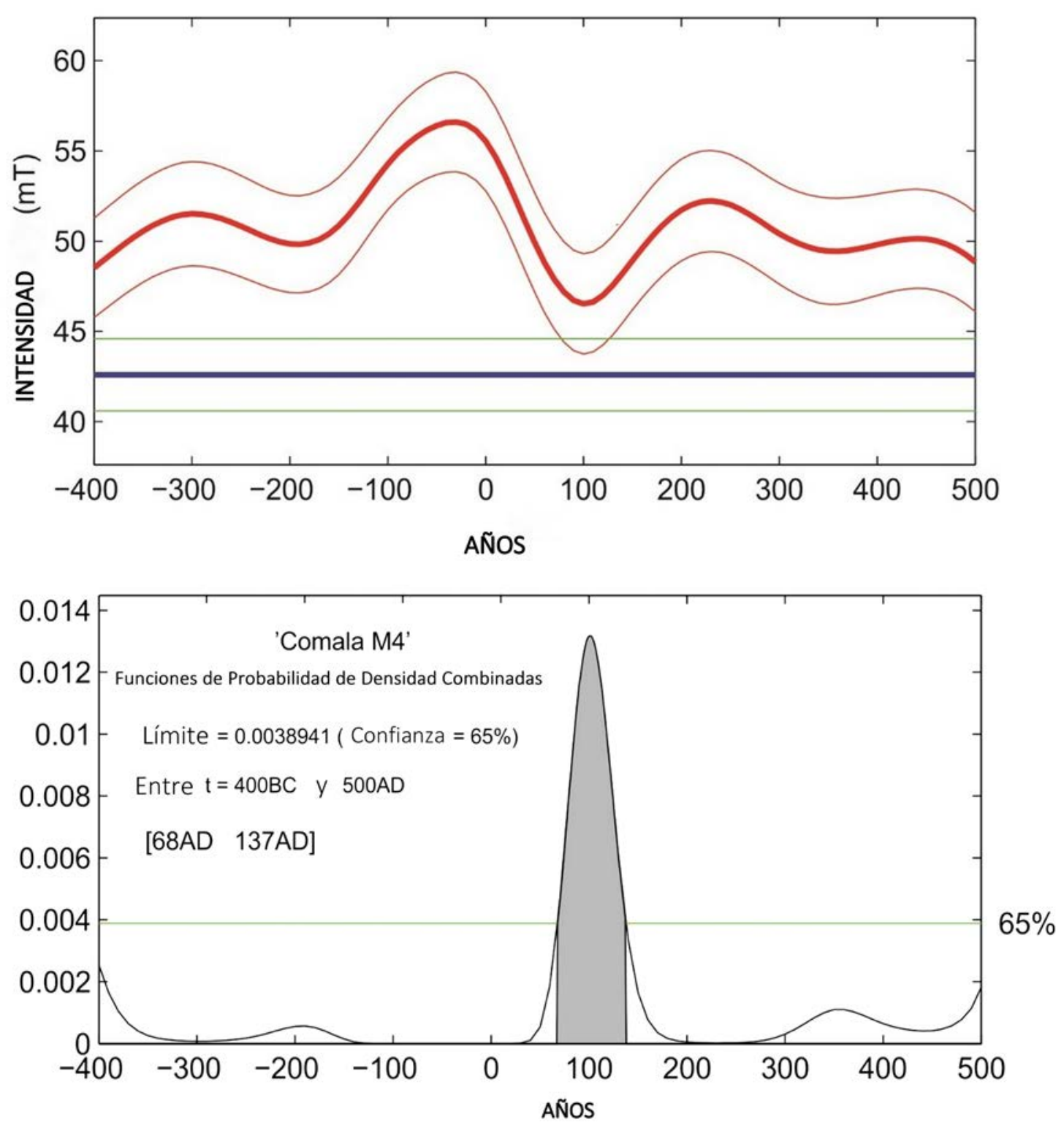

Ejemplo de fechamiento arqueomagnético para la muestra M4, usando la plataforma de Pavon-Carrasco et al. (2014).

valo más adecuado se basa en las consideraciones del contexto arqueológico o de la estratigrafía relativa, por lo cual es pertinente presentar por separado los datos obtenidos para cada sitio.

Para el sitio Comala se obtuvieron resultados para dos contextos:

- Un área de actividad al sur del círculo C, que pudo estar asociada con la celebración de banquetes, y que fue fechado a partir de dos muestras; M1 con un único intervalo entre 511-683 dC, el cual como ya se dijo no presenta ambigüedad dentro del intervalo elegido de datación; y la muestra M2, la cual tiene dos intervalos posibles entre 163-322 dC y 400-472 dC; consideramos que, dada la asociación estratigráfica de las muestras, el segundo intervalo de M2 sería el más adecuado.

- El relleno de la estructura 9 del círculo A fue fechado a partir de la muestra M 8, que presentó dos intervalos posibles entre 165-316 d.C., y 411-465 d.G., sin embargo, no existen elementos que permitan elegir uno u otro, por lo que se consideró el rango comprendido entre ambos. 
En cuanto al sitio Puente de Ladrillo, se obtuvieron tres intervalos de edades, asociados a las estructuras y patio del Círculo 1:

- La muestra M11 presentó dos intervalos posibles entre $132 \mathrm{aC}-34 \mathrm{dC}$, y 206-255 dC; dado que no hay criterios que permitan elegir uno, se consideró el rango comprendido entre ambos.

- La muestra M13 presentó solo un intervalo 597-839 dC, el cual no tiene ambigüedad dentro del intervalo elegido de datación.

- La muestra M3 se descartó, debido a que data del periodo Posclásico (entre el 1058 al 1453 dC), y dado que se recuperó en la capa I, consideramos que no corresponde a los materiales propios del sitio.

Por último, para el sitio La Herradura se obtuvo un intervalo correspondiente a los rellenos ubicados bajo la plaza circular: muestra M 4, con un único intervalo 68-137 dG, sin ambigüedad dentro del intervalo elegido de datación.

\section{Conclusiones}

Acorde a lo anterior, podemos decir que las edificaciones del círculo A y las áreas de actividad asociadas al círculo $\mathrm{C}$ del sitio Comala pueden corresponder al Clásico temprano (200 a 650 dC). Puente de Ladrillo pudo ser construido a finales de la fase Ortices ( $400 \mathrm{aC}$ a $100 \mathrm{dC}$ ) o inicios de la fase Comala (100 a $600 \mathrm{dC}$ ), pudiendo presentar una ocupación continua hacia el Clásico tardío (650 a $950 \mathrm{dC}$ ).

En el caso de La Herradura, es posible que la plaza y sus estructuras asociadas estuvieran en uso hacia el primer siglo de nuestra era, en los albores de la fase Comala (100 a $600 \mathrm{dC}$ ).

En suma, a través de los distintos intervalos obtenidos, es posible situar cronológicamente los sitios con plazas circulares en el valle de Colima, desde finales del Preclásico (400 aC a $200 \mathrm{dC}$ ) y hasta el Clásico temprano (200 a 650 dC); de forma particular, observamos que Puente de Ladrillo y La Herradura, tienen hasta ahora, evidencias mate- riales más tempranas que los estudiados para Comala, sin embargo, es menester contar con mucho más datos que permitan afinar las secuencias cronológicas de estos sitios y de la región.

Como vemos, los resultados obtenidos no contradicen los datos de Kelly, en tanto que funcionan como marco de referencia, sin embargo, consideramos necesario continuar con las dataciones por estos y otros métodos, con la finalidad de afinar la secuencia local y establecer límites más claros para las fases y aportar posibles correcciones a esta cronología.

\section{Referencias}

Abdalá, G., 2006, El urbanismo en el Occidente Mesoamericano. Sus determinantes geográficas y astronómicas dentro del área de influencia del volcán de Colima: México, Facultad de Arquitectura, Universidad Nacional Autónoma de México, Tesis Doctoral, 355 p.

Alcántara, S., 2005, Un panteón preclásico en Colima: México, Escuela Nacional de Antropología e Historia, Tesis de Licenciatura en Arqueología.

Alcántara, S., Galicia J., 2008, Aproximaciones al entendimiento de la cultura Capacha: Las Fuentes y Puertas de Rolón, dos casos de estudio, en $4^{\circ}$ Foro de Arqueología, Antropología e Historia de Colima, Colima, Secretaria de Cultura, Gobierno del estado de Colima.

Almendros L., 2012, El Periodo formativo en Colima: una continuidad ocupacional, Archivo técnico de la Coordinación Nacional de Arqueología.

Beekman, C., 2000, The Correspondence of Regional Patterns and Local Strategies in Formative to Classic Period West México: Journal of Anthropological Archaeology, 19(4), 385-412. 
Chauvin, A., Garcia, A., Lanos, P., Laubenheimer, F., 2000, Paleointensity of the geomagnetic field recovered on archeomagnetic sites from France: Physics of the Earth and Planetary Interiors, 120, 11-136.

Coe, R.S., 1967, Paleo-intensities of the Earth's magnetic field determined from Tertiary and Quaternary rocks: Journal of Geophysical Research, 72(12), 3247-3262.

Coe, R.S., Grommé, S., Mankinen, E.A., 1978, Geomagnetic paleointensities from radiocarbon-dated lava flows on Hawaii and the question of the Pacific nondipole low: Journal of Geophysical Research, Solid Earth: 83(B4), 1740-1756.

Crane, H.R., Griffin, J.B., 1972, University of Michigan Radiocarbon Dates XIV: Radiocarbon, 14(1), 155-94.

Kelly, I., 1980, Ceramic sequence in Colima: Capacha an early phase: Tucson, EUA, Antropological Papers, University of Arizona, 37, 110p.

Kelly, I., 1989, An archaeological reconnaissance of the West Coast: Nayarit to Michoacan: México, INAH, Homenaje a Isabel Kelly, Colección Científica, 179, 71-73.

Kosterov, A.A., Perrin, M., Glen, J.M., Coe, R.S., 1998, Paleointensity of the Earth's magnetic field in Early Cretaceous time: The Parana Basalt, Brazil: Journal of Geophysical Research, Solid Earth, 103(B5), 9739-9753.

Levi, S., 1977, The effect of magnetite particle size on paleointensity determinations of the geomagnetic field: Physics of the Earth and Planetary Interiors, 13(4), 245-259.

López, V., 2016, Aportaciones arqueomagnéticas a la cronología prehispánica del Occidente de México: Ciudad de México, Posgrado en Ciencias de la Tierra, Instituto de Geofísica, Universidad Nacional Autónoma de México, Tesis de Maestría, 186 p.
Morales, J., Goguitchaichvili, A., Acosta, G., Gonzalez-Moran, T., Alva-Valdivia, L., Robles-Camacho, J., HernandezBernal, M., 2009, Magnetic properties and archeointensity determination on pre-Columbian pottery from Chiapas, Mesoamerica: Earth, Planets and Space, 61(1), 83-91.

Morales,J., Goguitchaichvili, A., Olay, Á., Carvallo, C., Aguilar, B., 2013, Archeointensity investigation on pottery vestiges from Puertas de Rolón, Capacha culture: In search for affinity with other Mesoamerican preHispanic cultures: Studia Gephysica et Geodaetica, 57(4), 605-626.

Olay, M.A., Sánchez, L., 2015, La tradición Teuchitlán en Colima. Notas para una discusión: Revista Occidente, Revista Digital del Museo Nacional de Antropología, disponible en http://www.mna.inah.gob. $\mathrm{mx} /$ contexto.html.

Olay M.A., Platas R. R., Cabrera C. M.A. 2007, Informe Técnico Final del Salvamento Arqueológico La Herradura, Colima, México, Archivo Técnico de la Coordinación de Arqueología, INAH.

Olsson I., 2009, Radiocarbon dating history: early days, questions, and problems met: Radiocarbon, 15(1), 1-43.

Paterson, G.A., 2011, A simple test for the presence of multidomain behavior during paleointensity experiments: Journal of Geophysical Research, Solid Earth, 116(B10), B10104.

Pavon-Carrasco, FJ., Osete M.L., Torta J.M., De Santis A., 2014, A geomagnetic field model for the Holocene based on archaeomagnetic and lava flow data: Earth and Planetary Science Letters, 388, 98-109.

Sternberg, R., 1990, The geophysical basis of archaeomagnetic dating, en Eighmy J., 
Sternberg R. (eds.), Archaeomagnetic dating: Tucson, University of Arizona Press, 5-28.

Stuart, G., 2003, Pre-Hispanic Sociopolitical Development and Wetland Agriculture in the Tequila Valleys of West México: Arizona, EUA, Department of Anthropology, Arizona State University, Tesis doctoral, 645 p.

Thellier, E., Thellier, O., 1959, Sur l'intensité du champ magnétique terrestre dans le passé historique et géologique: Annales de Geophysique, 15, 285-376.

Weigand, P., 1985, Evidence for complex societies during the western Mesoamerican Classic period, en Foster, M., Weigand, P. (eds.), The archaeology of West and Northwest Mesoamerica: Colorado, Westview Press, 47-92.

Weigand, P., 1993, Evolución de una civilización prehispánica: arqueología de Jalisco, Nayarit y Zacatecas: Zamora, México, El Colegio de Michoacán, 444 p.

Weigand, P., 1996, The architecture of the Teuchitlan tradition of the Occidente of Mesoamerica: Ancient Mesoamerica, 7(1), 91-101.

Weigand, P., 2008, La tradición Teuchitlán del Occidente de México. Excavaciones en los Guachimontones de Teuchitlán, Jalisco, en Weigand, P., Beekman, C., Esparza, R. (eds.), Tradición Teuchitlán: México, El Colegio de Michoacán, Secretaría de Cultura del Estado de Jalisco, 29-62.

Weigand, P., Beekman C., 2008, Conclusiones: cronología y un intento de síntesis, en Weigand C., Beekman, C., Esparza R. (ed.), Tradición Teuchitlán: México, El Colegio de Michoacán, Secretaría de Cultura del Estado de Jalisco, 303-334. 